\title{
AtXRN4 Affects the Turnover of Chosen miRNA*s in Arabidopsis
}

\author{
Yan Liu ${ }^{1,2,+}$, Wenrui Gao ${ }^{1,3, \dagger}$, Shuangyang Wu ${ }^{1,2,+}{ }^{\circledR}$, Lu Lu ${ }^{1}$, Yaqiu Chen ${ }^{1,4}$, Junliang Guo ${ }^{1,5}$, \\ Shuzhen Men ${ }^{3}$ iD and Xiaoming Zhang ${ }^{1,2, *}$ \\ 1 State Key Laboratory of Integrated Management of Pest Insects and Rodents, Institute of Zoology, \\ Chinese Academy of Sciences, Beijing 100101, China; liuyan8882@126.com (Y.L.); \\ gaowenrui2020@126.com (W.G.); wushuangyang@ioz.ac.cn (S.W.); lulubio2018@163.com (L.L.); \\ 15670533706@163.com (Y.C.); guojunliang2008@163.com (J.G.) \\ 2 CAS Center for Excellence in Biotic Interactions, University of Chinese Academy of Sciences, \\ Beijing 100049, China \\ 3 Department of Plant Biology and Ecology, College of Life Sciences, Nankai University and Tianjin Key \\ Laboratory of Protein Science, Tianjin 300071, China; shuzhenmen@nankai.edu.cn \\ 4 Henan Normal University, Department of Life Sciences, Xinxiang, Henan 453007, China \\ 5 Institute of Physical Science and Information Technology, Anhui University, He fei, Anhui 230601, China \\ * Correspondence: zhangxm@ioz.ac.cn \\ + These authors contributed equally to this work.
}

Received: 13 January 2020; Accepted: 11 March 2020; Published: 13 March 2020

\begin{abstract}
Small RNA (sRNA) turnover is a key but poorly understood mechanism that determines the homeostasis of sRNAs. Animal XRN genes contribute the degradation of sRNAs, AtXRN2 and AtXRN3 also contribute the pri-miRNA processing and miRNA loop degradation in plants. However, the possible functions of the plant XRN genes in sRNA degradation are far from known. Here, we find that AtXRN4 contributes the turnover of plant sRNAs in Arabidopsis thaliana mainly by sRNA-seq, qRT-PCR and Northern blot. The mutation of AtXRN4 alters the sRNA profile and the accumulation of $21 \mathrm{nt}$ sRNAs was increased. Some miRNA*s levels are significantly increased in $x r n 4$ mutant plants. However, the accumulation of the primary miRNAs (pri-miRNAs) and miRNA precursors (pre-miRNAs) were generally unchanged in $x r n 4$ mutant plants which indicates that AtXRN4 contributes the degradation of some miRNA*s. Moreover, AtXRN4 interacts with Arabidopsis Argonaute 2 (AtAGO2). This interaction takes place in Processing bodies (P-bodies). Taken together, our observations identified the interaction between XRN4 with AtAGO2 and suggested that plant XRN4 also contributes the turnover of sRNAs.
\end{abstract}

Keywords: small RNA; RNA turnover; XRN; Argonaute; DCP

\section{Introduction}

Small RNAs (sRNAs) are 20-30 nucleotide (nt) noncoding RNAs that regulate the expression of target genes at the transcription or post-transcription levels [1,2]. The homeostasis of sRNAs is tightly regulated at the transcription and post-transcription levels [3,4]. Although the transcription and processing of microRNAs (miRNAs), a major sRNA for both animals and plants, have been well studied, the degradation mechanisms of miRNAs and other sRNAs remain unclear. The functions of exoribonucleases but not endoribonucleases have been proven in the turnover of sRNAs.

The turnover of sRNA is first demonstrated by its $3^{\prime}$ terminal modification and degradation. In plants, $3^{\prime}$ ends of sRNA duplexes are methylated by HUA Enhancer 1 (HEN1), which is a critical step in miRNA stabilization. The methylation of $3^{\prime}$ ends protects sRNAs from being degraded by $3^{\prime}$ truncation and uridylation [5]. HEN1 SUPPRESSOR1 (HESO1) and UTP: RNA Uridylyltransferase 1 
(URT1) act as $3^{\prime}$ uridylation transferases synergistically in the $3^{\prime}$ tailing [6,7]. Atrimmer 2 (ATRM2) acts in the $3^{\prime}-5^{\prime}$ degrading of unmethylated miRNA/miRNA* duplexes. Loss-of-function mutants of ATRM2 restored levels of a subset of miRNAs which were degraded in hen 1 mutants and they observed a marked increased trimming of several miRNA*s but not miRNAs in ATRM2 malfunction backgrounds which infers the existence of another yet unknown exoribonuclease [8]. SMALL RNA DEGRADING NUCLEASE (SDN) family were identified as $3^{\prime}-5^{\prime}$ exonucleases that degrade single-strand sRNAs in vivo and in vitro. SDN mutants accumulate higher levels of miRNAs and purified GST-SDN1 protein degrades single-strand sRNA in vitro with size of 17, 18, 20, 21, 22, 23, 24 and 27nt and yield an approximately 8-9 nt end products [9]. In animals, a number of $3^{\prime}-5^{\prime}$ exribonucleases have been reported to function in small RNA $3^{\prime}$ processing and degradation including MUT-7 and Poly(A)-specific Ribonuclease (PARN-1) in C. elegans, Nibbler in Drosophila [10,11]. RRP41degrades miR382 and some miRNAs in human embryonic kidney (HEK293T) cells [12]. ERI-1 degrades siRNA with 2-nt 3' overhangs [13]. The exoribonuclease DIS3L2 specifically binds and degrades uridylated RNA substrates such as uridylated pre-let-7, 7SL, and snRNA, but its homologous in Arabidopsis is inactive in Col-0 background.

The 5'-3' degradation of sRNAs has been partially studied in animals. The depletion of XRN1 and XRN2, two 5'-3' exoribonucleases encoded by Caenorhabditis elegans, leads increased accumulation of miRNAs and a subset of miRNA*s without the alterations of pri-miRNAs and pre-miRNAs. [14,15]. These observations indicate that CeXRN1 and CeXRN2 contribute to the degradation of sRNAs. Homo sapiens XRN1, but not HsXRN2, also contributes to the degradation of some miRNAs [12]. Recent studies showed that the accumulation of pri-miRNAs and corresponding mature miRNAs are increased and decreased in xrn2-1 respectively, which indicates that XRN2 promotes the processing of pri-miRNAs [16]. AtXRN2, AtXRN3, and AtXRN4 are the homologue genes encoded by Arabidopsis. AtXRN2 and AtXRN3 redundantly contribute the processing of pre-rRNA and the maturation of rRNA [17]. AtXRN4 is the closest homolog of yeast Xrn1p [18] AtXRN4, also referred to as EIN5, contributes the transduction of ethylene signal [19]. Function as a 5' $\rightarrow 3^{\prime}$ exoribonuclease, AtXRN4 is required for regulation of the F-box protein EBF1/2 which negatively regulate the key component of ethylene pathway, EIN3 [20,21]. Further study showed that ATXRN4 interacts EIN2 when it targets EBF1 3' UTR to cytoplasmic P-bodies [22]. AtXRN2, AtXRN3, and AtXRN4 are all involved in miRNA pathway. AtXRN2 and AtXRN3 eliminate the pri-miRNAs loop remnants after DCL1-mediated cleavage, while AtXRN4 contributes the degradation of the $3^{\prime}$ end of a subset of miRNA-mediated cleavage fragment $[17,23]$. Similar to miRNA-mediated decay in plants, posttranscriptional gene silencing (PTGS) is a process that involved RNA decay, small interfering RNA (siRNAs) and XRN activity [24]. The accumulations of decapped transgenic RNA and sRNAs were increased in ein 5 mutant plants [25]. Further study uncovered that EIN5 is a repressor for transgene and endogenous PTGS [26]. Upon the dysfunction of 5'-3' (EIN5) and 3'-5' (SKI-Exosome) RNA decay pathways, aberrant mRNAs are amplified by SGS3/RDR6 and processed into a large number of 21- to 22-nt endogenous siRNAs. These siRNAs are termed as coding transcripts derived small interfering RNA (ct-siRNA). However, the direct function of AtXRNs in the turnover of sRNAs is still unknown.

Argonaute (AGO) is the key component of RNA silencing [27]. Associated with sRNAs, AGO regulated the expression of sRNA target genes at transcriptional or post-transcriptional levels.

Arabidopsis encodes ten AGOs that are classified into three subgroups based on their sequence similarity: AGO1/5/10, AGO2/3/7, and AGO4/6/8/9 [28]. Most Arabidopsis miRNAs contain a $5^{\prime}$ terminal $\mathrm{U}$ and are predominantly loaded into AGO1 for function. In miRNA*s usually contain a $5^{\prime}$ terminal A and were predominantly loaded into AGO2 [29]. AGO2 shows both additive and overlapping activity with AGO1. Both of them contribute to plant defense against Pseudomonas and a broad range of viruses. Upon Pseudomonas infection, the loading of miR393b* into AGO2 was increased. miR393b* targets MEMB12 and the decreased accumulation of MEMB12 upon bacterial infection leads the increased secretion of antimicrobial protein PR1. Interestingly, the accumulation of miR393 is also increased 
upon bacterial infection and it corporately increase plant immunity by regulating auxin signaling pathway [30].

To detect the function of AtXRN4 in the sRNA degradation, we constructed sRNA libraries with Col-0 WT and xrn4 mutant plants. sRNA sequencing was then conducted with these sRNA libraries. The mutation of AtXRN4 altered the sRNA profile and the accumulations of miRNA*s was significantly increased in $x r n 4$ mutant plants. However, the accumulations of pri-miRNAs and pre-miRNAs were not generally altered which argue against the function of AtXRN4 in miRNA processing. These results indicated that AtXRN4 contributes to the turnover of some miRNA*s. Previous studies demonstrated that plant miRNA*s majorly associate with AtAGO2 $[29,30]$. We then determined the interaction of AtXRN4 with AtAGO2 and uncovered the association of these two proteins. Moreover, their interaction sites co-localize with the subcellular localization sites of DCP1, a marker protein of P-bodies. Our observations thus reveal the association between AtXRN4 with AtAGO2 and indicate that AtXRN4 affects the degradation of some Arabidopsis miRNA*s.

\section{Results}

\subsection{AtXRN4 Mutation Changes the sRNA Profiles}

Considering the significant roles of XRN genes in animal sRNA turnover, the role of AtXRN2 and AtXRN3 in pri-miRNA processing and miRNA loop degradation, and the key role of AtXRN4 in the degradation of a subset of 3' fragments of miRNA targets, we decided to determine the function of AtXRN in plant sRNA turnover. Our phylogenetic tree analysis of the $X R N$ orthologue proteins proved thatAtXRN2, AtXRN3, and AtXRN4 are all orthologs of CeXRN2rather than CeXRN1 (Figure S1). Moreover, AtXRN4 contains all the conserved exoribonuclease motifs (Figure S2) and showed a mild phenotype with serrated leaves (Figure S3b), multiple fruits emanate from the same node (Figure S3c), and late flowering (Figure S3d) which have described previously [24,31] Because of the important functions of AtXRN4 in mRNA degradation, we hypothesized that AtXRN4 may contribute to the turnover of plant sRNAs.

To determine the possible role of AtXRN4 in sRNA turnover, we first compared the sRNA population differences between Col-0 WT and xrn4 mutant plants. ein5-1, an EIN5 mutant that carries a "C" deletion at the fifteenth exon, was chosen for the small RNA profile analysis (Figure S3a) [20]. The AtXRN4 transcript was detected in Col-0 WT adult and Col-0 WT seedling plants (Figure S4). Moreover, the accumulation of the AtXRN4 transcript in rosette leaves was comparable to that in the rest of the tested tissues (Figure S4). Therefore, the four-week-old ein5-1 plants grown on the soil were collected to perform further sRNA deep sequencing analyses.

With total RNA isolated from collected tissues, two sets of sRNA libraries were constructed and sequenced. After removal of adapter sequences and low-quality reads, 18,762,306, 19,905,497, 18,650,051, and 19,905,780 clean sRNA reads were obtained from Col-0 WT and ein5-1 libraries, respectively (Figure S5a). There were 16,907,246, 18,037,364, 17,057,402, and 18,213,882 total sRNAs reads were mapped perfectly to the Arabidopsis genome for Col-0 WT and ein5-1, respectively. These sRNAs were clustered into 3,850,420, 4,154,868, 3,789,676, and 3,804,558 unique sRNA reads. The sample correlation matrix was evaluated with sRNA expression levels in the following color code: red represents strongly correlated and green represents weakly correlated. The matrix heatmap indicated that the sRNA libraries are highly reproducible (Figure S5b). Therefore, 4,002,644 and 3,797,117 average unique sRNAs were obtained for Col-0 WT and ein 5-1 mutant plants for further analysis, respectively.

The $5^{\prime}$-terminal nucleotide compositions of sRNAs from different libraries were analyzed first. Uridine $(\mathrm{U})$ or adenosine $(\mathrm{A})$ are the dominant $5^{\prime}$-terminal nucleotides for both Col-0 WT and ein 5-1 libraries (Figure 1a). Moreover, the 5'-terminal nucleotide compositions of sRNAs in Col-0 WT and ein5-1 libraries were comparable (Figure 1a). Next, the size distributions of sRNAs were analyzed. In the ein5-1 mutants, 21nt sRNAs increased, while $24 \mathrm{nt}$ sRNAs decreased, which demonstrated that the malfunction of AtXRN4 alters the length distributions of plant sRNAs (Figure 1b). 

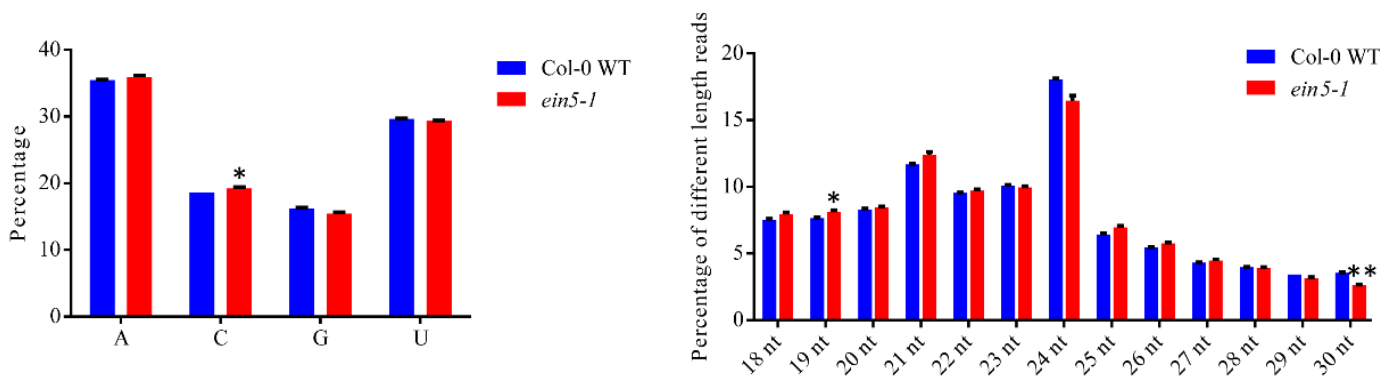

Figure 1. AtXRN4 mutants change the sRNA profiles. (a) $5^{\prime}$-terminal nucleotide frequency of sRNAs in Col-0 WT and ein5-1 mutant plants. (b) Size distribution of sRNAs in Col-0 WT and ein5-1 mutant plants. One star means $\mathrm{P}<0.05$, two star means $\mathrm{P}<0.01$, three star means $\mathrm{P}<0.001$, four star means $\mathrm{P}<0.0001$.

\subsection{AtXRN4 Decreases the Accumulation of miRNA*s}

Due to the increased PTGS, a previous study showed that 21 and 22nt siRNAs derived from aberrant coding transcript (ct-siRNAs) accumulated in ein5/ski2 double mutants [26]. Indeed, the $22 \mathrm{nt}$ sRNAs in ein5-1 plants were also slightly increased over those in Col-0 WT plants (Figure 1b). This so slight increase probably because of the small percentage of ct-siRNA. However, the enhancement of $21 \mathrm{nt}$ sRNAs was more abundant than that of the $22 \mathrm{nt}$ sRNAs. Therefore, the accumulation of some other $21 \mathrm{nt}$ sRNAs may also be increased in ein5-1 mutant plants.

To determine the $21 \mathrm{nt}$ sRNAs that were enhanced in ein5-1 mutants, the 5'-terminal nucleotide compositions of $21 \mathrm{nt}$ sRNAs were analyzed. The $5^{\prime}$-terminal nucleotide compositions of $22 \mathrm{nt}$ sRNAs were analyzed as controls. " $\mathrm{U}$ " and " $\mathrm{A}$ " were the dominant 5 '-terminal nucleotides for both $21 \mathrm{nt}$ and $22 \mathrm{nt}$ sRNAs. The accumulation of $21 \mathrm{nt}$ sRNAs containing 5 '-terminal " $\mathrm{A}$ ", " $\mathrm{C}$ ", " $\mathrm{G}$ " was significantly increased in ein 5-1 mutant plants. The accumulation of 22nt sRNA containing 5 '-terminal " $\mathrm{C}$ " is also increase in ein5-1 mutant plants while the $22 \mathrm{nt}$ sRNAs start " $\mathrm{A}$ " and " $\mathrm{G}$ " are comparable in Col-0 WT and ein5-1 mutant plants (Figure 2a,b). 5'-terminal preference bias is different for 21- and 22-nt sRNA, which is consistent with the published results [29,32,33]. Most miRNA*s associated with AtAGO2 have a 5'-terminal "A" $[29,30]$. The accumulations of miRNA*s were thus analyzed. Indeed, miRNA*s increased in ein5-1 mutant plants (Figure 2c). Meanwhile, ct-siRNAs and miRNAs increased

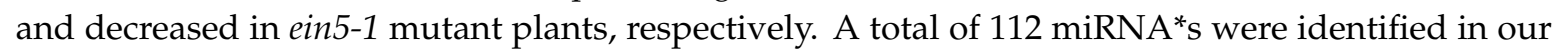
sRNA libraries, and the accumulation of 88 of them was higher than 2 reads (or at least 1 reads in each sample) (Table S1). To further determine the profile of miRNA*s, we performed mean abundance plot analysis of these miRNA*s. Among the 88 miRNA*s analyzed, 22 miRNA*s in the ein5-1 mutants were more than 1.5 times higher than those in Col-0 WT plants (Figure $2 \mathrm{~d}$ and Table S1). In contrast, only 6 miRNA*s were down-regulated in ein $5-1$ mutants. Therefore, AtXRN4 affects the accumulation of a subset of miRNA*s.

qRT-PCR and Northern blot assays were then conducted to further validate the role of AtXRN4 on miRNA* accumulation. ein5-1 and ein5-6, another AtXRN4 mutant that contains a T-DNA insertion at the fifth exon, were used to perform qRT-PCR experiments (Figure S3a). The 14 most abundant miRNA*s that were up-regulated in ein5-1 mutant plants (ratio > 1.5) were selected for qRT-PCR experiments (Table S1). Indeed, the accumulation of all miRNA*s tested in ein5-1 mutant plants was significantly higher than that in Col-0 WT plants (Figure 2e, right panel). The accumulations of 18 unchanged miRNA*s (ratio $<1.5$ ) were also determined by qRT-PCT. The accumulation of most of these miRNA*s was also increased in ein5-1 and ein5-6 mutants (Figure 2e, left panel). Consistent with the sRNA sequencing assays, the enhancements of most candidates in the left parts of Figure 2e were lower than those in the right parts of Figure 2e. The four most significantly increased miRNA*s, miR169f*/miR169e*, miR396a*/miR396b*, miR398b*, and miR168a* were selected for Northern blot analysis (Figure S6, miR408* is undetectable by Northern blot). The accumulations of miR393b*, miR391b*, and miR472*, the three abundant miRNA*s with weaker increases, were also tested. As 
shown in Figure $2 \mathrm{f}$, the accumulations of miR472*, miR396b*, miR168a*, miR398b*, and miR169f* in ein5-1 and ein5-6 were much higher than those in Col-0 WT plants. In contrast, the accumulations of miR393b* and miR391* were slightly higher than those in control plants. We also determined the corresponding miRNA expression by qRT-PCR and Northern blot, the results showed that the accumulations of a subset of miRNAs corresponding to the highly increased miRNA*s also increased in ein 5-1 mutant plants (Figure S7). Therefore, EIN5 may alter the stability of miRNA/miRNA* duplexes. Altogether, these results indicated that AtXRN4 decreases the accumulation of a subset of miRNA*s and miRNAs.
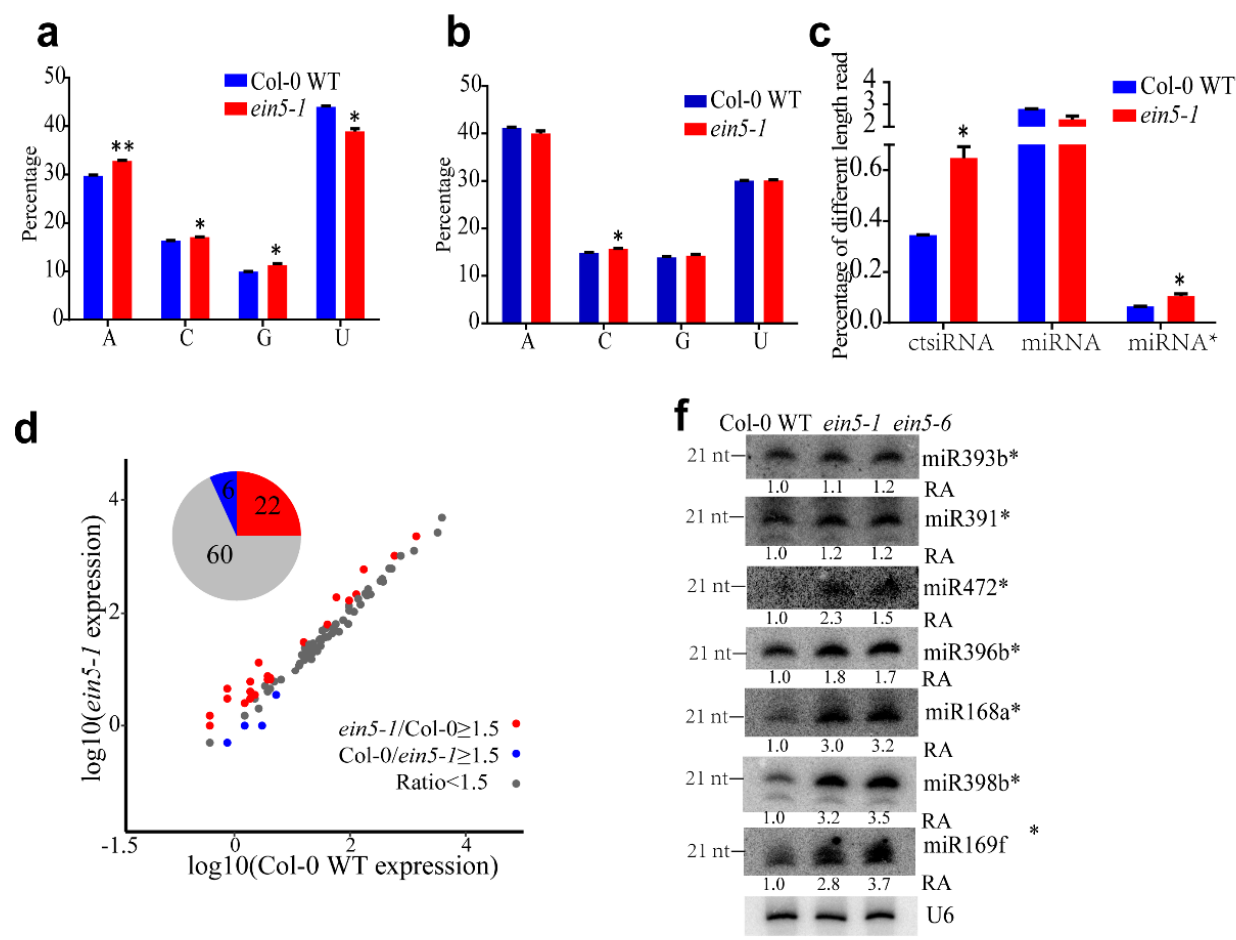

e

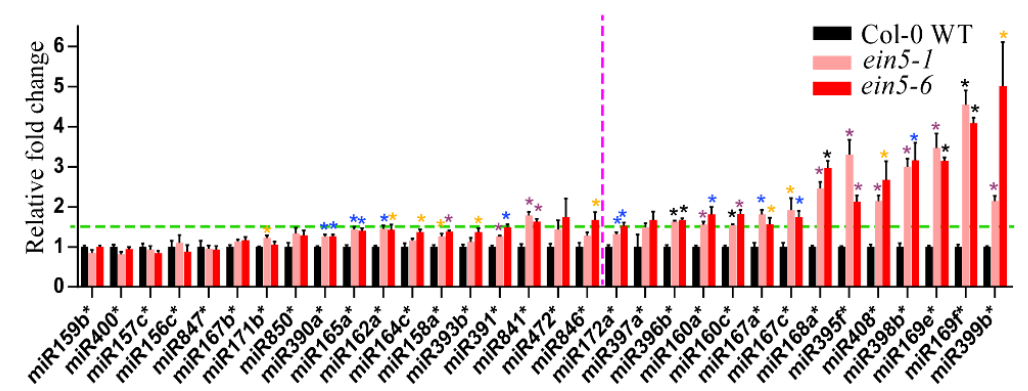

Figure 2. AtXRN4 decreases the accumulation of miRNA*s.(a) The $5^{\prime}$-terminal nucleotide frequency of $21 \mathrm{nt}$ sRNAs in Col-0 WT and ein5-1 mutant plants. (b) The 5'-terminal nucleotide frequency of $22 \mathrm{nt}$ sRNAs in Col-0 WT and ein5-1 mutant plants. (c) The relative accumulation of different $21 \mathrm{nt}$ sRNAs in Col-0 WT and ein5-1 mutant plants. (d) Mean abundance plot analysis of miRNA*s in Col-0 WT and ein5-1 mutant plants. miRNA*s with mean reads over 2 are shown. miRNA*s with significant changes (ein5-1/Col-0 WT $\geq 1.5$ and Col-0 WT/ein5-1 $\geq 1.5$ ) are highlighted with red and blue dots, respectively. (e) qRT-PCR validation of miRNA*s in different plants. The relative expression of 14 miRNA*s with significant increases ( $>1.5$-fold) and 18miRNA*s without significant changes are shown in the right and left panels, respectively. The green line indicates the 1.5-fold change. qRT-PCR data are shown as the means \pm SEM. Yellow star means $\mathrm{P}<0.05$, blue star means $\mathrm{P}<0.01$, purple star means $\mathrm{P}<0.001$, black star means $\mathrm{P}<0.0001$. Similar results were obtained in more than three biological repeats. (f) Northern blot was used to detect the accumulation of miRNA*s in different plants. U6 served as the loading control. Similar results were obtained in three biological repeats. Primers and probes are listed in Table S2. 


\subsection{AtXRN4 does not Alter theTranscription and Processing of miRNAsPrecursors}

The abundance of sRNA is a balance between the biogenesis and turnover of sRNAs. In plants, the pri-miRNAs transcribed by RNA Pol II were processed by DCL1 into pre-miRNAs, which were further processed into short double stranded RNAs (miRNA/miRNA*) [34,35].

The possible functions of AtXRN4 on miRNA* transcription was first evaluated. The accumulations of corresponding pri-miRNAs were detected using qRT-PCR. The accumulations of pri-miR391 in ein5-6 and pri-miR472 and pri-miR398in ein5-1/ein5-6 mutant plants were higher than those in Col-0 WT plants (Figure 3a). However, the accumulations of the remaining pri-miRNAs in ein5-1 and ein5-6 mutant plants were comparable to those in Col-0 WT plants. Portion of the pri-miRNA* tested are higher than those in the Col-0 WT plants. However, most of the accumulation of tested miRNA*s in ein5 mutants are higher than those in the Col-0 WT plants. Similar results were obtained in fry1 mutant [36]. Moreover, the transcription and processing of pri-miRNAs take place in nuclear, while XRN4 localizes inP-bodies [22,37]. These results argue against the general role of XRN4 on the accumulation of miRNA*s caused by the increased accumulation of pri-miRNAs. To test the possible role of AtXRN4 on miRNA* processing, we performed Northern blot analysis to detect the accumulations of corresponding pre-miRNAs. No differences in pre-miRNA accumulations were observed among Col-0 WT, ein5-1, and ein5-6, which argued against a role of AtXRN4 in miRNA* precursor processing (Figure 3b). Taken together, these results indicated that AtXRN4 decreased miRNA* accumulation downstream of the miRNA precursors processing step.

\section{a}

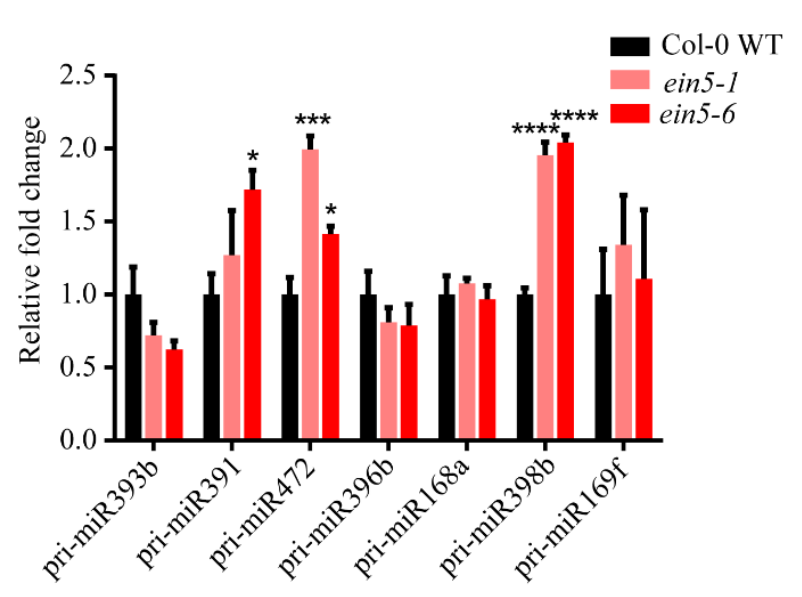

b

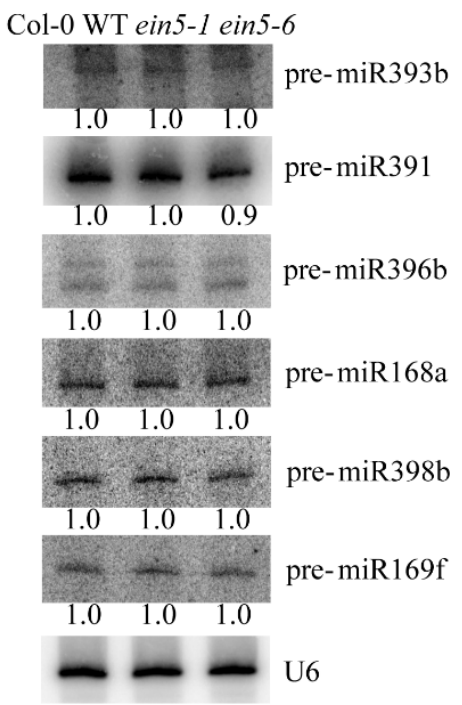

Figure 3. AtXRN4 does not affect the transcription and processing of miRNA*s. (a) qRT-PCR analysis of pri-miRNAs in Col-0 WT and $x r n 4$ mutant plants. Actin2 was used as an internal control. One star means $\mathrm{P}<0.05$, two star means $\mathrm{P}<0.01$, three star means $\mathrm{P}<0.001$, four star means $\mathrm{P}<0.0001$. (b)Northern blot analysis of the corresponding precursors of miRNA*s in Col-0 WT and $x r n 4$ mutant plants. U6 was used as the loading control. Probes are listed in Table S2. Similar results were obtained in three biological repeats.

\subsection{AtXRN2 and AtXRN3 do not Alter the Accumulations of Corresponding miRNA*s}

AtXRN2 and AtXRN3 are also XRN homologues in Arabidopsis, the functions of them in the accumulation of these miRNA*s were then determined. Two-week-old $x r n 2-1, x r n 3-2$ and $x r n 2-1 / x r n 3-2$ seedlings were collected to perform qRT-PCR. ein5-1 and ein5-6 seeding was involved as control and the accumulations of corresponding miRNA*s were also increased in ein5-1 seedling. However, the accumulations of these miRNA*s were not significantly altered in $x r n 2-1, x r n 3-2$ and $x r n 2-1 / x r n 3-2$ seedlings (Figure 4). We also determined the corresponding miRNA expression by qRT-PCR, the 
results showed that the accumulations of a subset of miRNAs corresponding to the highly increased miRNA*s also increased in ein5-1 mutant plants (Figure S8). Taken together, these results showed that only AtXRN4 contributes the accumulation of miRNA*s.

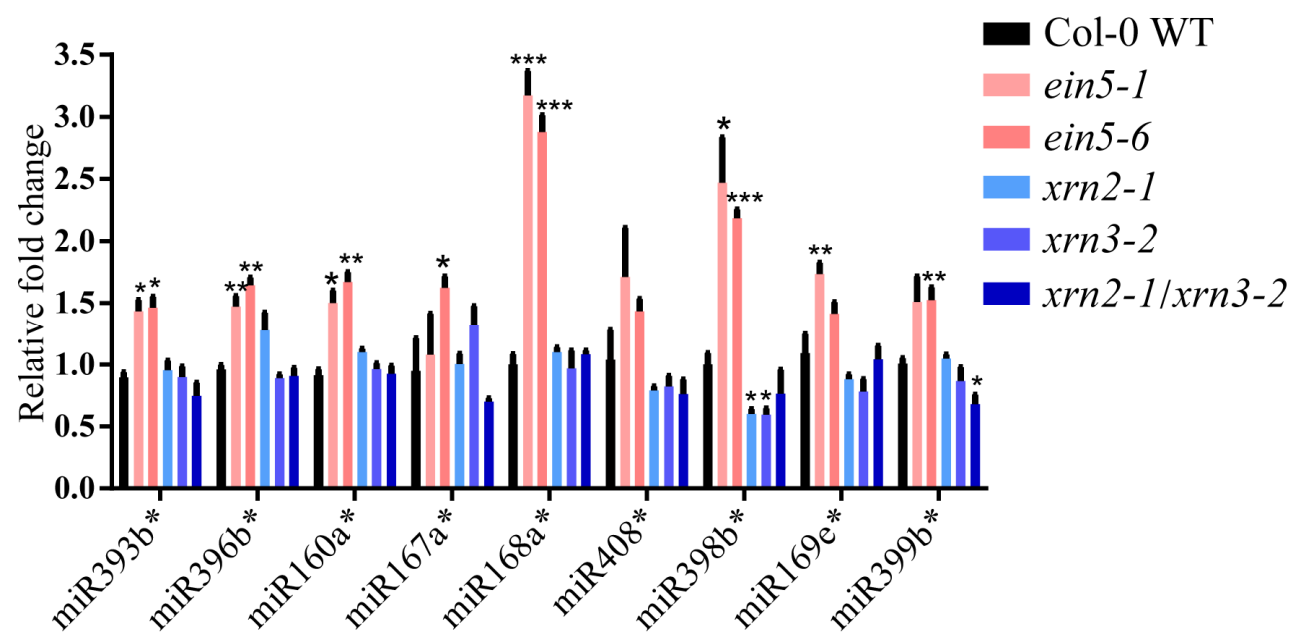

Figure 4. AtXRN2 and AtXRN3 do not decrease the accumulation of corresponding miRNA*s. qRT-PCR analysis of these accumulated miRNA*s in $x r n 2-1, x r n 3-2$ and $x r n 2-1 x r n 3-2$ mutants seedlings. Col-0 WT, ein5-1 and ein5- 6 were involved as control. One star means $\mathrm{P}<0.05$, two star means $\mathrm{P}<0.01$, three star means $\mathrm{P}<0.001$, four star means $\mathrm{P}<0.0001$.

\subsection{AtXRN4 Interacts with AtAGO2 in P-Bodies}

After processing, miRNA/miRNA* duplexes are loaded into AtAGO, the core component of the RNA-induced silencing complex (RISC) for maturation, regulation and degradation [38]. Our results showed that AtXRN4 affects the turnover but not the transcription and processing of miRNA*s, indicating that the function of AtXRN4 may correlate with the function of AtAGO.

AtAGO1 is the major AtAGO associated with miRNAs while AtAGO2 is the major AtAGO associated with miRNA*s. miRNA*s especially these with $5^{\prime}$ terminal A were predominantly associated with AGO2 [29,30,39]. Therefore, AtXRN4 may interact with AtAGO2 and affect miRNAs and miRNA*s turnover. Firstly, we predicted we predicted the co-expression gene of AtAGO2 at http: //atted.jp/ (a plant co-expression database) and find AtXRN4 in the co-expression list. To confirm the interaction possibility, a co-immunoprecipitation (Co-IP) assay was conducted in Nicotianabenthamiana. HA-tagged AtAGO2 was co-expressed with FLAG-tagged AtXRN4 and with FLAG-tagged MEMB12 as a negative control [30]. With the $\alpha$-HA antibody, HA-AtAGO2 was easily detected in AtXRN4-FLAG IP fractions but not in MEMB12-FLAG IP fractions (Figure 5a). To further confirm this interaction, a bimolecular fluorescence complementation (BiFC) assay was conducted. Both nYFP-AtAGO2 and AtXRN4-cYFP were co-expressed in N. benthamiana. In addition, nYFP-GST and cYFP-GST were co-inoculated as negative controls. Strong fluorescence was detected in nYFP-AtAGO2 and AtXRN4-cYFP co-expression plants but not in control plants (Figure 5b). These results proved the interaction between AtXRN4 and AtAGO2.

The YFP signal accumulates in punctate compartments in nYFP-AtAGO2 and AtXRN4-cYFP co-expression plants (Figure 5b). P-bodies display punctate structures in the cytoplasm, and AtXRN4has been reported to co-localize with DCP1 and DCP2, the marker proteins of P-bodies [37]. Therefore, AtXRN4 may interact with AtAGO2 in P-bodies. We thus co-inoculated nYFP-AtAGO2 and AtXRN4-cYFP with DCP1-RFP to test this possibility. The punctate structures of YFP signals largely overlapped with the RFP signals (Figure 5c). Taken together, AtXRN4 interacts with AtAGO2 in P-bodies. 
a

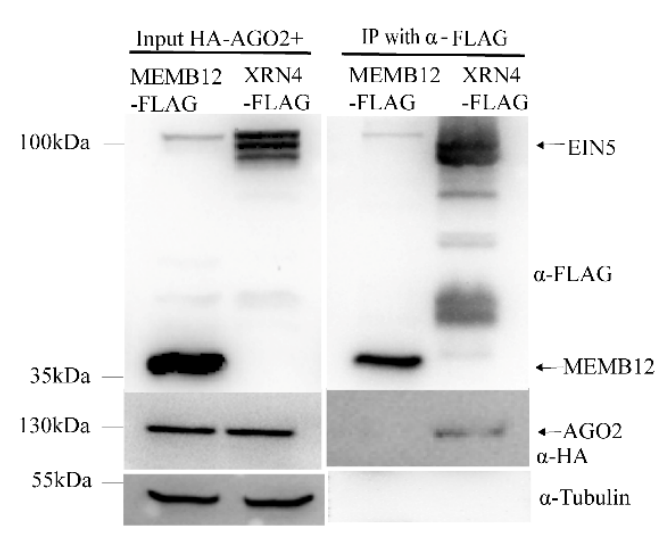

b

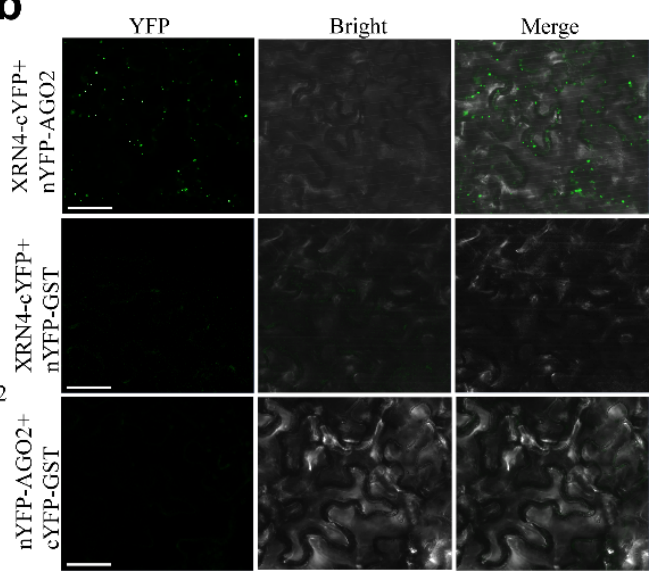

C

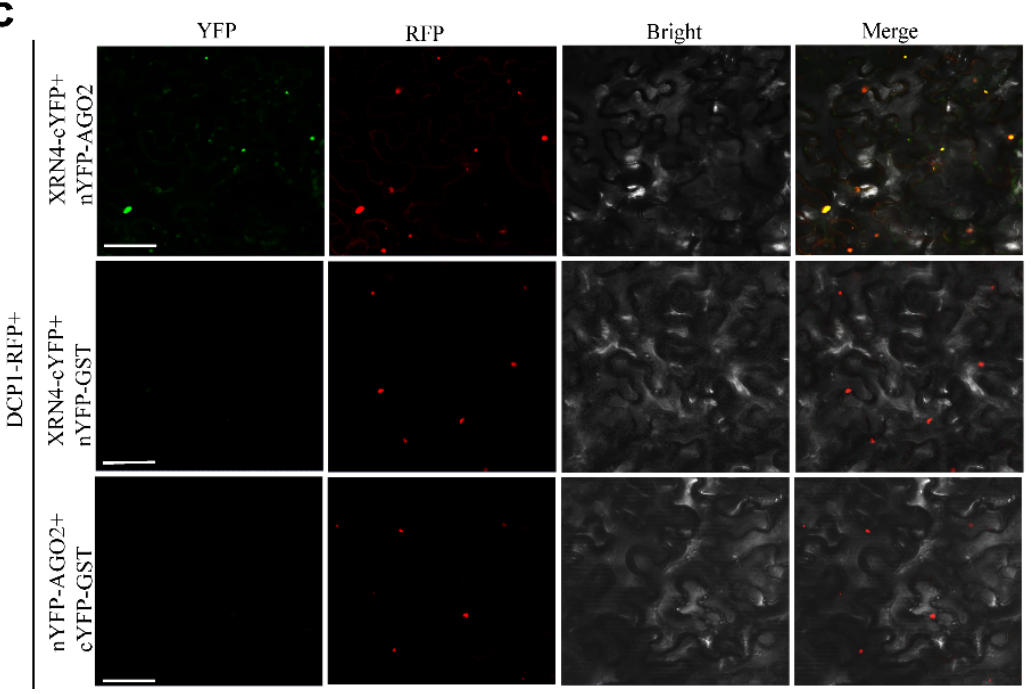

Figure 5. AtXRN4 interacts with AtAGO2 in P-bodies. (a) Co-IP experiment in N. benthamiana shows that AtXRN4 interacts with AtAGO2. IP was conducted using the $\alpha$-FLAG antibody. Input and IP products were probed with anti-HA and anti-FLAG antibodies, respectively. (b) BiFC analysis of the interaction between AtXRN4 and AtAGO2. YFP images were taken 48 hpi. Scale bars, $50 \mu \mathrm{m}$. (c) AtXRN4-AtAGO2 interaction sites colocalize with DCP1, a marker protein for P-bodies. Green fluorescence indicates YFP and red fluorescence indicates DCP1-RFP. Scale bar, $50 \mu \mathrm{m}$.

\section{Discussion}

sRNA turnover is a key but poorly understood mechanism that determines the accumulation of sRNAs $[3,11]$. The plant exoribonuclease that conducts 5'-3' sRNA degradation has not been identified. In this study, we found that AtXRN4, a 5'-3' exoribonuclease in Arabidopsis, is involved in the turnover of miRNA*s. We then revealed that AtXRN4 interacts with AtAGO2, an AtAGO that primarily loads miRNA*s, in P-bodies which play a fundamental role in RNA decay. Therefore, the function of AtXRN4 in miRNA* degradation may correlate with the function of AtAGO2.

Our observations demonstrate that AtXRN4, a 5'-3' exoribonuclease in plants, contributes to the turnover of plant miRNA*s. XRN genes play key roles in the turnover of different RNAs in eukaryotes $[40,41]$. In the nucleus, DmXRN2 degrades mRNAs that are decapped by LSM2-8 proteins to restrict their localization in the nucleus [42]. HsXRN2 also degrades the unprotected $5^{\prime}$ end of transcripts processed from mRNA to promote transcription termination [43]. In the cytoplasm, XRN1 degrades decapped mRNA in yeast, humans, flies and worms [42,44-46]. The role of XRN in sRNA degradation was first identified in C. elegans. Depletion of both CeXRN1 and CeXRN2 increased the accumulation of miRNAs or miRNA*s without altering the accumulation of pre-miRNAs $[14,15]$. 
HsXRN1, but not HsXRN2, was found to contribute to the degradation of miR382 and a subset of other miRNA*s [12]. Plants lack an XRN1 orthologue but encode three orthologs of XRN2, AtXRN2, AtXRN3, and AtXRN4 [18]. AtXRN4 degrades endogenous mRNAs and decapped transgene mRNAs [23,25]. Although the degradation of mRNA transcripts by AtXRN4 inhibits the initiation of RNA silencing of endogenous genes and transgenes [25,26], the function of AtXRN on sRNA degradation has not been determined. Here, we found that the mutation of AtXRN4 leads to the increase accumulation of a subset of miRNA*s (Figure 2) without alternations in the corresponding pri-miRNAs and pre-miRNAs (Figure 3). These results indicate that AtXRN4 is a plant $5^{\prime}-3^{\prime}$ exoribonuclease that conducts the turnover of sRNAs.

The accumulation of rRNA-derived sRNA loaded into AtAGO1 were increased in $x r n 2-1 / x r n 3-2$ mutant plants and it leads the decreased accumulation of un-protected miRNAs [36]. However, the accumulations of test miRNA*s were not significantly altered in $x r n 2-1 / x r n 3-2$ mutant seedling (Figure 4), which is consistent with the association of miRNAs but not miRNA*s with AtAGO1 [29]. Therefore, distinct XRN genes may regulate the accumulation of different sRNAs.

The degradation of plant miRNA*s may occur in P-bodies. XRN1 co-localizes with DCP1 and DCP2, the proteins that conduct the decapping process, in P-bodies and performs mRNA degradation in yeast [47]. DCS-1, a decapping scavenger enzyme, interacts with CeXRN1 and promotes the degradation of miRNAs in C. elegans [48]. The human orthologue of DCS-1 is also required for the 5'-3' exoribonuclease function of XRN2 and plays key roles in miRNA degradation [49]. As a marker protein for the 5'-3' mRNA degradation pathway, AtXRN4 has been reported to co-localize with DCP1 in P-bodies [37]. AGO1, AGO2 and reporter mRNA that are targeted by miRNAs were also reported to localize in P-bodies [50]. P-bodies have been found to link to RNAi (RNA interference) and miRNAs mediated decay and translational repression [50-53]. Here, we proved that AtXRN4 interacts with AtAGO2, a major AtAGO that associates with miRNA*s, in P-bodies (Figure 5) [29,30,39]. ATRM2, a 3'-5' exoribonuclease that degrades unmethylated miRNA/miRNA*s, also interacts with AtAGO1 in punctate structures in the cytoplasm [8]. These results suggest that the P-body is an organelle that degrades both mRNAs and sRNAs. AtXRN4 is likely to directly or indirectly regulate these miRNA*s in P-bodies. The interaction of AtXRN4 with AtAGO2 indicates that the function of AtXRN4 on sRNAs may correlate with AtAGO2 in P-bodies.

AtXRN4 only contributes to the turnover of a subset of miRNA*s. CeXRN1 and CeXRN2 contribute to the degradation of a subset of miRNAs in C. elegans $[14,15,54]$. The accumulation of a subset of miRNA*s with thermodynamic asymmetry also increased in Cexrn1 and Cexrn2 malfunction tissues, indicating that CeXRN1 and CeXRN2 degrade some miRNA*s [14]. HsXRN1 also only contributes to the degradation of miR382 and some other miRNAs [12]. This specific instability is derived from their 3'-terminal sequences, and a mutation in the seven 3'-terminal nucleotides of miR382 increases its stability. ATRM2, the $3^{\prime}-5^{\prime}$ exoribonuclease in plants, also affects the accumulation of a subset of unmethylated miRNA/miRNA* duplexes [8]. However, how ATRM2 fulfills this selection bias is unknown. The accumulation of some miRNA*s is altered differently in Atxrn4 mutant plants, which indicates that AtXRN4 may also degrade a subset of miRNA*s. In Atxrn4 mutants, the up-regulated miRNA*s have different $5^{\prime}$ ends. Therefore, the sRNA characteristics that determine the selection bias of AtXRN4 need to be further determined.

As an important component of ethylene signal pathway, AtXRN4 interacts with EIN2 to targets EBF13' UTR to cytoplasmic P-body [22]. AtXRN4 mutants showed ethylene insensitive phenotype [20]. Ethylene has been found to be key factors for fruit ripening, seed germination, sexual determination of flower, root nodulation, and response to pathogens and stresses [55]. The effect of AtXRN4 malfunction on miRNA pathway may be the result on ethylene signaling pathway. This possibility cannot be ruled out and deserves further study. 


\section{Materials and Methods}

\subsection{Plant Materials and Growth Conditions}

Plants were grown in a green house at $22{ }^{\circ} \mathrm{C}, 70 \%$ humidity and a $12-\mathrm{h}$ light/12-h dark photoperiod. ein5-1 [19] and ein5-6 [20] mutant plants have been isolated by Dr. Joseph R. Ecker's group previously. These seeds are kindly gifts from Dr. Hongwei Guo and Dr. Menji Cao. xrn2-1, xrn3-2 and xrn2-1/xrn3-2 seeds are kindly gifts from Dr. Beixin Mo [36]. The ein5-1, ein5-6, xrn2-1 and xrn3-2 mutant seeds are also available on TAIR database (www.arabidopsis.org).

\subsection{RNA Extraction and sRNASequencing}

Total RNA isolation and library construction were performed as described previously [33]. Briefly, four-week-old plants were collected, and RNA was isolated with TRIzol (Invitrogen, Carlsbad, CA, USA,) according to the manufacturer's instructions. Approximately $1 \mathrm{~g}$ rosette leaves were ground in liquid nitrogen and mixed with $10 \mathrm{~mL}$ TRIzol for RNA isolation. After thoroughly mixing, 1/2 volume chloroform was added. The solution was then vortexed thoroughly and put on ice for $5 \mathrm{~min}$. Then it was centrifuge at $12000 \mathrm{rpm}$ and $4{ }^{\circ} \mathrm{C}$ for $15 \mathrm{~min}$. The supernatant was mixed with 2.2 -fold volume of ethanol and stored at $-20{ }^{\circ} \mathrm{C}$ overnight. The mix was then centrifuge at $12000 \mathrm{rpm}$ and $4{ }^{\circ} \mathrm{C}$ for $15 \mathrm{~min}$. The pellet was washed with $75 \%$ ethanol and finally dissolved with RNase-free $\mathrm{H} 2 \mathrm{O}$. The concentration and quality of the isolated RNA were determined by a NanoDrop spectrophotometer (Thermo Scientific, Waltham, MA, USA) and gel electrophoresis, respectively. Total RNA (20 $\mu \mathrm{g})$ was used for sRNA library construction with the Illumina TruSeq sRNA Library Preparation Kit (Illumina, San Diego, CA, USA). 15-30nt sRNAs were cut from the gel for library construction. TwosRNA libraries were constructed for both Col-0 WT and ein5-1 samples. sRNA sequencing was performed with an Illumina HiSeq 2500 instrument.

\section{3. sRNA Sequencing Analysis}

We used fast for removing adapter and filtering low-quality reads [56]. Bowtie was used to map reads to Arabidopsis genome with specific parameters (bowtie -f -v 1 -p 10 -a -m 1 -best -strata) [57]. Correlation was calculated using Euclidean's distance matrix using PtR program in Trinity package [58]. Reads count was also calculated by BEDTOOLS with unique mapping reads. BLAT was used to find the best hit of miRNA* in the genome [59]. BEDTOOLS was used to intersect the locus between miRNA and miRNA* in order to make sure the miRNA-miRNA* pair in the same transcript [60]. Differential gene expression analyses of replicated count data were carried out using the R package EdgeR [61]. The reads count of miRNA*s from different samples were normalized by the number of total tRNA reads as performed in previous study [62].

\section{4. $q R T-P C R$}

For sRNA qRT-PCR, $1 \mu \mathrm{g}$ of total RNA was digested with DNase I (Takara, Dalian, China, and poly(A) was added to the $3^{\prime}$ end by E. coli poly(A) polymerase (NEB). The first-strand cDNAs were transcribed by the Moloney murine leukemia virus (M-MuLV) reverse transcriptase (NEB). qRT-PCR was performed in a 96-well plate with TB GreenTM Premix Ex TaqTM (TIi RNase H Plus) (Takara, Dalian, China). The $10 \mu \mathrm{l}$ PCRcontained $5 \mu \mathrm{l}$ 2x TB Green ${ }^{\mathrm{TM}}$ Premix Ex Taq ${ }^{\mathrm{TM}}, 2 \mu \mathrm{cDNA}$ template (diluted 1/10-fold before use) and $200 \mathrm{nM}$ primers. Every sRNA forward primers were listed in Table S2 and all reverse primers are $3^{\prime}$ race inner listed in Table S2. The PCR was performed as follows: $95^{\circ} \mathrm{C}$ for $30 \mathrm{~s}$ and 40 cycles of $95^{\circ} \mathrm{C}$ for $5 \mathrm{~s}$ and $60{ }^{\circ} \mathrm{C}$ for $30 \mathrm{~s}$. U6 was used as an endogenous control.

For mRNA qRT-PCR, $1 \mu \mathrm{g}$ of total RNA was used for reverse transcription according to the manufacturer's instructions with the PrimeScript ${ }^{\mathrm{TM}}$ RT reagent Kit with a gDNA Eraser (Takara, Dalian, China) and the random primer were used in the kit. The PCR was the same as the sRNA qRT-PCR process except for primers which were also listed in Table S2. Actin2 was used as the control. The 
relative fold change in the expression levels were calculated using the $2^{-\Delta \Delta C t}$ method. All reactions were carried out in two technical repeats and four biological repeats.

\subsection{Northern Blot}

A total of $60 \mu \mathrm{g}$ total RNA was loaded onto a $14 \%$ denaturing urea-polyacrylamide gel and run with $0.5 \mathrm{x}$ TBE at $150 \mathrm{~V}$. The gel was transferred to Amersham HybondTM-NX with 14A overnight. Chemical cross-linking buffer was prepared as follows: $0.373 \mathrm{~g} \mathrm{~N}$-(3-Dimethylaminopropyl)$\mathrm{N}^{\prime}$-ethylcarbodiimide hydrochloride (Sigma-Aldrich, E7550, St. Louis, Missouri, MI, USA), 3 drops of $1 \mathrm{M} \mathrm{HCl}, 121 \mu \mathrm{l}$ 1-Methylimidazole (Sigma, M50834), and $12 \mathrm{~mL}$ DEPC-treated ddH2O. Membrane was putted on a plate which has two layers of filter paper that was soaked in cross-linking buffer, then the plate was covered by plastic film andincubatedat $60{ }^{\circ} \mathrm{C}$ for $2 \mathrm{~h}$ and at $85{ }^{\circ} \mathrm{C}$ for another $2 \mathrm{~h}$. The probes were labeled with $\gamma$-P32 ATP by T4-polynucleotide kinase (NEB). Pre-miRNA Northern blot probes are the same as miRNA Northern blot probes. The membrane was pre-incubated with PerfectHyb ${ }^{\mathrm{TM}}$ Plus Hybridization Buffer liquid (Sigma) for 30min, then the labeled probe was added and incubated for $12 \mathrm{~h}$. After that, the membrane was wash with buffer contains 2x SSC and $0.025 \%$ SDS for four times. Signal was collected with phosphor screen and scanned with typhoon. Similar results were obtained from three biological repeats.

\subsection{Plasmid Construction}

To perform the Co-IP assay, XRN4, MEMB12 and AtAGO2 were cloned into the pENTR vector (Invitrogen, Carlsbad, CA, USA, K2420-020) and then recombined into the Gateway destination vectors pEarlyGate202 (pEG202), pFH, and pMDC32 [63-65] by the LR reaction with the LR enzyme (Invitrogen, Carlsbad, CA, USA, 11791-020).

To conduct the BiFC assay, the GST fragment was cloned into the pENTR vector. GST, XRN4, and AtAGO2 fragments were then cloned into pSITE-cEYFP or pSITE-nEYFP vector by the LR reaction.

For the co-localization assay, the DCP1 fragment was cloned into the pENTR vector. The fragment was then cloned into pEarlyGate102 (pEG102) by the LR reaction.

\subsection{BiFC and Colocalization Assay}

XRN4-pSITE-cEYFP-AtXRN4, pSITE-nEYFP-AtAGO2, pSITE-nEYFP-GST and pSITE-cEYFP-GST constructs were transformed into the Argobacterium strain GV3101. Argobacterium were then infiltrated into N. benthamiana with an OD600nm $=1.0$ as described before [66]. Fluorescence was determined at 72hourspost-inoculation(hpi) with a Zeiss LSM-710 confocal microscope (Carl Zeiss, Thornwood, NY, USA). YFP fluorescence was excited at $514 \mathrm{~nm}$.

For the co-localization analysis, XRN4-pSITE-cEYFP-AtXRN4 and pSITE-nEYFP-AtAGO2 were co-inoculated with pEG102-DCP1-RFP in N. benthamiana. YFP and RFP fluorescence were excited at $514 \mathrm{~nm}$ and $561 \mathrm{~nm}$, respectively. Similar results were obtained from more than three biological replicates.

\subsection{Co-IP}

pEG202-AtXRN4 and pMDC32-AtAGO2 were co-infiltrated into four-week-old N. benthamiana. Samples were collected at $72 \mathrm{hpi}$. MEMB12-pFH was used as a negative control. Leaves were ground with liquid nitrogen and extracted with $15 \mathrm{~mL}$ extraction buffer $(20 \mathrm{mM}$ Tris- $\mathrm{HCl}(\mathrm{pH} 7.5), 300 \mathrm{mM}$ $\mathrm{NaCl}, 5 \mathrm{mM} \mathrm{MgCl} 2,5 \mathrm{mM}$ dithiothreitol [DTT], 0.5\% TWEEN-20, and 1 tablet of complete protease inhibitor (Roche)). After centrifugation (5000 rpm, $15 \mathrm{~min}$ ), the supernatants were incubated with FLAG beads (Sigma Aldrich, USA, A2220) for $4 \mathrm{~h}$. Then, the beads were washed three times with washing buffer ( $150 \mathrm{mM} \mathrm{NaCl}, 5 \mathrm{mM}$ DTT, $5 \mathrm{mM} \mathrm{MgCl}$, 0.3\% Triton X-100, $20 \mathrm{mM}$ Tris- $\mathrm{HCl}$ (pH 7.5), and 1 pellet per $50 \mathrm{~mL}$ Complete EDTA-free protease inhibitor (Roche)). Monoclonal anti-HA-HRP (Santa Cruz Biotechnology, sc7392), monoclonal anti-FLAG-HRP (Sigma, A8592) and monoclonal 
anti- $\alpha$-tubulin (Sigma, T6074) antibodies were used for Western blot. Similar results were obtained from three biological replicates

\section{Conclusions}

Taken together, our observations indicate that AtXRN4 contributes to the turnover of sRNAs. AtXRN4 interacts with AtAGO2 and may degrade sRNAs in P-bodies. Our results provide new insight into plant sRNAs homeostasis and further studies needed to be performed to determine its function mechanism.

Supplementary Materials: The following are available online at http://www.mdpi.com/2223-7747/9/3/362/s1, Table S1: miRNA*s in Col-0 WT and ein5-1 plants. Table S2: Primers and probes used in the study. Figure S1: Phylogenetic tree analysis of XRN homolog proteins in 12 species. Figure S2: Alignment of the A. thaliana XRN4 protein with orthologs from Ce, C. elegans; Hs, H. sapiens; Sc, S. cerevisiae. Figure S3. xrn4 mutant plants display mild developmental phenotype similar to observed before. Figure S4: The relative expression of AtXRN4 in different tissues and stages in Col-0 WT plants. Figure S5: Summary of sRNA sequencing data and heatmap of the Pearson correlation between the expression levels of sRNA in Col-0 WT and ein5-1 mutant plants. Figure S6: Difference analysis of miRNA* accumulations between two plants. Figure S7: miRNA expression level. Figure S8: miRNA expression level in two-week seedling of ein5, xrn2 and $x r n 3$ mutants.

Author Contributions: X.Z. initiated the project; X.Z. and Y.L. designed the experiments; Y.L., W.G., J.G. and Y.C. conducted the experiments; S.W. and L.L. performed the bioinformatic analyses; X.Z., Y.L., and S.M. wrote the paper. All authors have read and approved the manuscript for publication.

Funding: This work is supported by the National Key Plan for Scientific Research and Development of China (2017YFD0200904 and 2017YFD0200406), Ministry of Agriculture Transgenic Major Projects (2016ZX08010001), Strategic Priority Research Program of the CAS (No. XDB11050700), National Natural Science Foundation of China (NSFC 91954105, 31622046, 91540116, 31700233, 31900224, 31872302, and 31471782), Beijing Municipal Natural Science Foundation (6192021, 5202017), Program of CAS(ZDBS-LY-SM027), and Open Research Fund Program of State Key Laboratory of Integrated Pest Management (ChineseIPM1715).

Acknowledgments: We thank Hongwei Guo, Songnian Hu, Jianing Geng, Beixin Mo and Mengji Cao for providing materials.

Conflicts of Interest: The authors declare no conflict of interest.

Availability of Data and Materials: The sRNA deep sequencing data has been deposited in the Sequence Read Archive (SRA) database with Bioproject accession number PRJNA587899.

\section{References}

1. Huang, J.; Yang, M.; Zhang, X. The function of small RNAs in plant biotic stress response. J. Integr. Plant Biol. 2016, 58, 312-327. [CrossRef]

2. Borges, F.; Martienssen, R.A. The expanding world of small RNAs in plants. Nat. Rev. Mol. Cell Biol. 2015, 16, 727. [CrossRef]

3. Sanei, M.; Chen, X. Mechanisms of microRNA turnover. Curr. Opin. Plant Biol. 2015, 27, 199-206. [CrossRef]

4. Rüegger, S.; Großhans, H. MicroRNA turnover: When, how, and why. Trends Biochem. Sci. 2012, 37, 436-446. [CrossRef] [PubMed]

5. Li, J.; Yang, Z.; Yu, B.; Liu, J.; Chen, X. Methylation protects miRNAs and siRNAs from a 3'-end uridylation activity in Arabidopsis. Curr. Biol. CB 2005, 15, 1501-1507. [CrossRef] [PubMed]

6. Ren, G.; Chen, X.; Yu, B. Uridylation of miRNAs by hen1 suppressor1 in Arabidopsis. Curr. Biol. CB 2012, 22, 695-700. [CrossRef] [PubMed]

7. Wang, X.; Zhang, S.; Dou, Y.; Zhang, C.; Chen, X.; Yu, B.; Ren, G. Synergistic and independent actions of multiple terminal nucleotidyl transferases in the $3^{\prime}$ tailing of small RNAs in Arabidopsis. PLoS Genet. 2015, 11, e1005091. [CrossRef]

8. Wang, X.; Wang, Y.; Dou, Y.; Chen, L.; Wang, J.; Jiang, N.; Guo, C.; Yao, Q.; Wang, C.; Liu, L.; et al. Degradation of unmethylated miRNA/miRNA*s by a DEDDy-type 3' to 5' exoribonuclease Atrimmer 2 in Arabidopsis. Proc. Natl. Acad. Sci. USA 2018, 115, E6659-E6667. [CrossRef]

9. Ramachandran, V.; Chen, X. Degradation of microRNAs by a family of exoribonucleases in Arabidopsis. Science 2008, 321, 1490-1492. [CrossRef] 
10. Tops, B.B.; Tabara, H.; Sijen, T.; Simmer, F.; Mello, C.C.; Plasterk, R.H.; Ketting, R.F. RDE-2 interacts with MUT-7 to mediate RNA interference in Caenorhabditis elegans. Nucleic Acids Res. 2005, 33, 347-355. [CrossRef]

11. Wang, J.; Mei, J.; Ren, G. Plant microRNAs: Biogenesis, homeostasis, and degradation. Front. Plant Sci. 2019, 10, 360. [CrossRef] [PubMed]

12. Bail, S.; Swerdel, M.; Liu, H.; Jiao, X.; Goff, L.A.; Hart, R.P.; Kiledjian, M. Differential regulation of microRNA stability. RNA 2010, 16, 1032-1039. [CrossRef] [PubMed]

13. Thomas, M.F.; L'Etoile, N.D.; Ansel, K.M. Eri1: A conserved enzyme at the crossroads of multiple RNA-processing pathways. Trends Genet. TIG 2014, 30, 298-307. [CrossRef] [PubMed]

14. Chatterjee, S.; Fasler, M.; Büssing, I.; Großhans, H. Target-mediated protection of endogenous microRNAs in C. elegans. Dev. Cell 2011, 20, 388-396. [CrossRef] [PubMed]

15. Chatterjee, S.; Großhans, H. Active turnover modulates mature microRNA activity in Caenorhabditis elegans. Nature 2009, 461, 546-549. [CrossRef] [PubMed]

16. Fang, X.; Zhao, G.; Zhang, S.; Li, Y.; Gu, H.; Li, Y.; Zhao, Q.; Qi, Y. Chloroplast-to-nucleus signaling regulates microRNA biogenesis in Arabidopsis. Dev. Cell 2019, 48, 371-382.e4. [CrossRef]

17. Kurihara, Y. Activity and roles of Arabidopsis thaliana XRN family exoribonucleases in noncoding RNA pathways. J. Plant Res. 2017, 130, 25-31. [CrossRef]

18. Kastenmayer, J.; Green, P. Novel features of the XRN-family in Arabidopsis: Evidence that AtXRN4, one of several orthologs of nuclear Xrn2p/Rat1p, functions in the cytoplasm. Proc. Natl. Acad. Sci. USA 2000, 97, 13985-13990. [CrossRef]

19. Roman, G.; Lubarsky, B.; Kieber, J.J.; Rothenberg, M.; Ecker, J.R. Genetic analysis of ethylene signal transduction in Arabidopsis thaliana: Five novel mutant loci integrated into a stress response pathway. Genetics 1995, 139, 1393-1409.

20. Olmedo, G.; Guo, H.; Gregory, B.D.; Nourizadeh, S.D.; Aguilar-Henonin, L.; Li, H.; An, F.; Guzman, P.; Ecker, J.R. ETHYLENE-INSENSITIVE5 encodes a 5' $\rightarrow 3^{\prime}$ exoribonuclease required for regulation of the EIN3-targeting F-box proteins EBF1/2. Proc. Natl. Acad. Sci. USA 2006, 103, 13286-13293. [CrossRef]

21. Potuschak, T.; Vansiri, A.; Binder, B.M.; Lechner, E.; Vierstra, R.D.; Genschik, P. The exoribonuclease XRN4 is a component of the ethylene response pathway in Arabidopsis. Plant Cell 2006, 18, 3047-3057. [CrossRef]

22. Li, W.; Ma, M.; Feng, Y.; Li, H.; Wang, Y.; Ma, Y.; Li, M.; An, F.; Guo, H. EIN2-directed translational regulation of ethylene signaling in Arabidopsis. Cell 2015, 163, 670-683. [CrossRef] [PubMed]

23. Souret, F.F.; Kastenmayer, J.P.; Green, P.J. AtXRN4 degrades mRNA in Arabidopsis and its substrates include selected miRNA targets. Mol. Cell 2004, 15, 173-183. [CrossRef] [PubMed]

24. Nagarajan, V.K.; Jones, C.I.; Newbury, S.F.; Green, P.J. XRN 5' $\rightarrow 3^{\prime}$ exoribonucleases: Structure, mechanisms and functions. Biochim. Biophys. Acta 2013, 1829, 590-603. [CrossRef] [PubMed]

25. Gazzani, S.; Lawrenson, T.; Woodward, C.; Headon, D.; Sablowski, R. A link between mRNA turnover and RNA interference in Arabidopsis. Science 2004, 306, 1046-1048. [CrossRef] [PubMed]

26. Zhang, X.; Zhu, Y.; Liu, X.; Hong, X.; Xu, Y.; Zhu, P.; Shen, Y.; Wu, H.; Ji, Y.; Wen, X.; et al. Suppression of endogenous gene silencing by bidirectional cytoplasmic RNA decay in Arabidopsis. Science 2015, 348, 120-123. [CrossRef]

27. Bohmert, K.; Camus, I.; Bellini, C.; Bouchez, D.; Caboche, M.; Benning, C. AGO1 defines a novel locus of Arabidopsis controlling leaf development. EMBO J. 1998, 17, 170-180. [CrossRef]

28. Bologna, N.G.; Voinnet, O. The diversity, biogenesis, and activities of endogenous silencing small RNAs in Arabidopsis. Annu. Rev. Plant Biol. 2014, 65, 473-503. [CrossRef]

29. Mi, S.; Cai, T.; Hu, Y.; Chen, Y.; Hodges, E.; Ni, F.; Wu, L.; Li, S.; Zhou, H.; Long, C.; et al. Sorting of small RNAs into Arabidopsis argonaute complexes is directed by the $5^{\prime}$ terminal nucleotide. Cell 2008, 133, 116-127. [CrossRef]

30. Zhang, X.; Zhao, H.; Gao, S.; Wang, W.C.; Katiyar-Agarwal, S.; Huang, H.D.; Raikhel, N.; Jin, H. Arabidopsis Argonaute 2 regulates innate immunity via miRNA393 $\left(^{*}\right)$-mediated silencing of a Golgi-localized SNARE gene, MEMB12. Mol. Cell 2011, 42, 356-366. [CrossRef]

31. Gregory, B.D.; O’Malley, R.C.; Lister, R.; Urich, M.A.; Tonti-Filippini, J.; Chen, H.; Millar, A.H.; Ecker, J.R. A link between RNA metabolism and silencing affecting Arabidopsis development. Dev. Cell 2008, 14, 854-866. [CrossRef] [PubMed]

32. Sun, F.; Guo, G.; Du, J.; Guo, W.; Peng, H.; Ni, Z.; Sun, Q.; Yao, Y. Whole-genome discovery of miRNAs and their targets in wheat (Triticum aestivum L.). BMC Plant Biol. 2014, 14, 142. [CrossRef] [PubMed] 
33. Yang, M.; Xu, Z.; Zhao, W.; Liu, Q.; Li, Q.; Lu, L.; Liu, R.; Zhang, X.; Cui, F. Rice stripe virus-derived siRNAs play different regulatory roles in rice and in the insect vector Laodelphax striatellus. BMC Plant Biol. 2018, 18, 219. [CrossRef] [PubMed]

34. Margis, R.; Fusaro, A.F.; Smith, N.A.; Curtin, S.J.; Watson, J.M.; Finnegan, E.J.; Waterhouse, P.M. The evolution and diversification of Dicers in plants. FEBS Lett. 2006, 580, 2442-2450. [CrossRef]

35. Liu, C.; Axtell, M.J.; Fedoroff, N.V. The helicase and RNaseIIIa domains of Arabidopsis Dicer-Like1 modulate catalytic parameters during microRNA biogenesis. Plant Physiol. 2012, 159, 748-758. [CrossRef]

36. You, C.; He, W.; Hang, R.; Zhang, C.; Cao, X.; Guo, H.; Chen, X.; Cui, J.; Mo, B. FIERY1 promotes microRNA accumulation by suppressing rRNA-derived small interfering RNAs in Arabidopsis. Nat. Commun. 2019, 10, 4424. [CrossRef]

37. Weber, C.; Nover, L.; Fauth, M. Plant stress granules and mRNA processing bodies are distinct from heat stress granules. Plant J. Cell Mol. Biol. 2008, 56, 517-530. [CrossRef]

38. Rogers, K.; Chen, X. Biogenesis, turnover, and mode of action of plant microRNAs. Plant Cell 2013, 25, 2383-2399. [CrossRef]

39. Montgomery, T.A.; Howell, M.D.; Cuperus, J.T.; Li, D.; Hansen, J.E.; Alexander, A.L.; Chapman, E.J.; Fahlgren, N.; Allen, E.; Carrington, J.C. Specificity of ARGONAUTE7-miR390 interaction and dual functionality in TAS3 trans-acting siRNA formation. Cell 2008, 133, 128-141. [CrossRef]

40. Houseley, J.; Tollervey, D. The many pathways of RNA degradation. Cell 2009, 136, 763-776. [CrossRef]

41. Meyer, S.; Temme, C.; Wahle, E. Messenger RNA turnover in eukaryotes: Pathways and enzymes. Crit. Rev. Biochem. Mol. Biol. 2004, 39, 197-216. [CrossRef] [PubMed]

42. Till, D.D.; Linz, B.; Seago, J.E.; Elgar, S.J.; Marujo, P.E.; de Lourdes Elias, M.; Arraiano, C.M.; McClellan, J.A.; McCarthy, J.E.; Newbury, S.F. Identification and developmental expression of a $5^{\prime}-3^{\prime}$ exoribonuclease from Drosophila melanogaster. Mech. Dev. 1998, 79, 51-55. [CrossRef]

43. West, S.; Gromak, N.; Proudfoot, N.J. Human 5' $\rightarrow 3^{\prime}$ exonuclease Xrn2 promotes transcription termination at co-transcriptional cleavage sites. Nature 2004, 432, 522. [CrossRef] [PubMed]

44. Stevens, A. Purification and characterization of a Saccharomyces cerevisiae exoribonuclease which yields 5'-mononucleotides by a 5'leads to 3'mode of hydrolysis. J. Biol. Chem. 1980, 255, 3080-3085. [PubMed]

45. Bashkirov, V.I.; Scherthan, H.; Solinger, J.A.; Buerstedde, J.-M.; Heyer, W.-D. A mouse cytoplasmic exoribonuclease (mXRN1p) with preference for G4 tetraplex substrates. J. Cell Biol. 1997, 136, 761-773. [CrossRef] [PubMed]

46. NEWBURY, S.; Woollard, A. The 5'-3' exoribonuclease xrn-1 is essential for ventral epithelial enclosure during, C. elegans embryogenesis. RNA 2004, 10, 59-65. [CrossRef]

47. Sheth, U.; Parker, R. Decapping and decay of messenger RNA occur in cytoplasmic processing bodies. Science 2003, 300, 805-808. [CrossRef]

48. Bossé, G.D.; Rüegger, S.; Ow, M.C.; Vasquez-Rifo, A.; Rondeau, E.L.; Ambros, V.R.; Großhans, H.; Simard, M.J. The decapping scavenger enzyme DCS-1 controls microRNA levels in Caenorhabditis elegans. Mol. Cell 2013, 50, 281-287. [CrossRef]

49. Meziane, O.; Piquet, S.; Bossé, G.D.; Gagné, D.; Paquet, E.; Robert, C.; Tones, M.A.; Simard, M.J. The human decapping scavenger enzyme DcpS modulates microRNA turnover. Sci. Rep. 2015, 5, 16688. [CrossRef]

50. Liu, J.; Valencia-Sanchez, M.A.; Hannon, G.J.; Parker, R. MicroRNA-dependent localization of targeted mRNAs to mammalian P-bodies. Nat. Cell Biol. 2005, 7, 719-723. [CrossRef]

51. Pauley, K.M.; Eystathioy, T.; Jakymiw, A.; Hamel, J.C.; Fritzler, M.J.; Chan, E.K. Formation of GW bodies is a consequence of microRNA genesis. EMBO Rep. 2006, 7, 904-910. [CrossRef] [PubMed]

52. Detzer, A.; Engel, C.; Wunsche, W.; Sczakiel, G. Cell stress is related to re-localization of Argonaute 2 and to decreased RNA interference in human cells. Nucleic Acids Res. 2011, 39, 2727-2741. [CrossRef] [PubMed]

53. Wang, X.; Chang, L.; Wang, H.; Su, A.; Wu, Z. Dcp1a and GW182 induce distinct cellular aggregates and have different effects on microRNA pathway. DNA Cell Biol. 2017, 36, 565-570. [CrossRef] [PubMed]

54. Miki, T.S.; Rüegger, S.; Gaidatzis, D.; Stadler, M.B.; Großhans, H. Engineering of a conditional allele reveals multiple roles of XRN2 in Caenorhabditis elegans development and substrate specificity in microRNA turnover. Nucleic Acids Res. 2014, 42, 4056-4067. [CrossRef]

55. Guo, H.; Ecker, J.R. The ethylene signaling pathway: New insights. Curr. Opin. Plant Biol. 2004, 7, 40-49. [CrossRef] 
56. Chen, S.; Zhou, Y.; Chen, Y.; Gu, J. FASTP: An ultra-fast all-in-one FASTQ preprocessor. Bioinformatics 2018, 34, i884-i890. [CrossRef]

57. Langmead, B.; Trapnell, C.; Pop, M.; Salzberg, S.L. Ultrafast and memory-efficient alignment of short DNA sequences to the human genome. Genome Biol. 2009, 10, R25. [CrossRef]

58. Haas, B.J.; Papanicolaou, A.; Yassour, M.; Grabherr, M.; Blood, P.D.; Bowden, J.; Couger, M.B.; Eccles, D.; Li, B.; Lieber, M. De novo transcript sequence reconstruction from RNA-seq using the Trinity platform for reference generation and analysis. Nat. Protoc. 2013, 8, 1494. [CrossRef]

59. Kent, W.J. BLAT-The BLAST-like alignment tool. Genome Res. 2002, 12, 656-664. [CrossRef]

60. Quinlan, A.R.; Hall, I.M. BEDTools: A flexible suite of utilities for comparing genomic features. Bioinformatics 2010, 26, 841-842. [CrossRef]

61. Robinson, M.D.; McCarthy, D.J.; Smyth, G.K. edgeR: A Bioconductor package for differential expression analysis of digital gene expression data. Bioinformatics 2010, 26, 139-140. [CrossRef] [PubMed]

62. Zhang, Z.; Hu, F.; Sung, M.W.; Shu, C.; Castillo-González, C.; Koiwa, H.; Tang, G.; Dickman, M.; Li, P.; Zhang, X. RISC-interacting clearing 3'-5' exoribonucleases (RICEs) degrade uridylated cleavage fragments to maintain functional RISC in Arabidopsis thaliana. eLife 2017, 6, e24466. [CrossRef] [PubMed]

63. Curtis, M.D.; Grossniklaus, U. A gateway cloning vector set for high-throughput functional analysis of genes in planta. Plant Physiol. 2003, 133, 462-469. [CrossRef] [PubMed]

64. Earley, K.W.; Haag, J.R.; Pontes, O.; Opper, K.; Juehne, T.; Song, K.; Pikaard, C.S. Gateway-compatible vectors for plant functional genomics and proteomics. Plant J. Cell Mol. Biol. 2006, 45, 616-629. [CrossRef]

65. Zanetti, M.E.; Chang, I.F.; Gong, F.; Galbraith, D.W.; Bailey-Serres, J. Immunopurification of polyribosomal complexes of Arabidopsis for global analysis of gene expression. Plant Physiol. 2005, 138, 624-635. [CrossRef]

66. Zhang, X.; Niu, D.; Carbonell, A.; Wang, A.; Lee, A.; Tun, V.; Wang, Z.; Carrington, J.C.; Chang, C.E.; Jin, H. ARGONAUTE PIWI domain and microRNA duplex structure regulate small RNA sorting in Arabidopsis. Nat. Commun. 2014, 5, 5468. [CrossRef]

(C) 2020 by the authors. Licensee MDPI, Basel, Switzerland. This article is an open access article distributed under the terms and conditions of the Creative Commons Attribution (CC BY) license (http://creativecommons.org/licenses/by/4.0/). 